\title{
Continuous smoking cessation was associated with weight gain
}

\author{
Klesges RC, Winders SE, Meyers AW, et al. How much weight gain occurs following smoking cessation? A comparison of weight gain \\ using both continuous and point prevalence abstinence. J Consult Clin Psychol 1997 Apr;65:286-91.
}

\section{Objective}

To determine the effects of continuous and point prevalence smoking cessation on weight gain, and the characteristics of people at risk of weight gain after cessation.

\section{Design}

12 month cohort study of volunteers who participated in a cognitive behavioural smoking cessation study group.

\section{Setting \\ USA.}

\section{Participants}

196 participants (mean age 44 years, 62\% women) who completed all 8 group sessions; attended 1 month, 6 month, and 12 month follow up sessions; and met criteria for classification into 1 of 3 smoking status groups: continuously smoking (self reported smoking or carbon monoxide [CO] concentrations $\geqslant$ 10 parts per million [ppm] at each follow up) $(\mathrm{n}=118)$; continuously abstinent (no self reported smoking and CO concentrations $<10 \mathrm{ppm}$ at each follow up) $(\mathrm{n}=51)$; or point prevalent abstinent (abstinent at $1 \mathrm{y}$, no self reported smoking in the past 24 hours, CO concentrations $<10 \mathrm{ppm}$, self reports of not being regular smokers, and self reported smoking or CO concentrations $\geqslant 10 \mathrm{ppm}$ at one or both of the previous follow ups) $(\mathrm{n}=27)$.

\section{Assessment of risk factors}

Baseline data were collected on sex, race, age, marital status, level of education, total family income, number of cigarettes smoked per day, and number of years as a regular smoker. Participants also completed a 10 item questionnaire about restrained eating practices. Self reported smoking confirmed by CO testing and weight were recorded at each follow up session.

\section{Main outcome measure}

Weight gain (weight at follow up minus weight at baseline).

\begin{abstract}
Main results
At 12 months, the mean weight gain for people in the continuously abstinent, point prevalence, and continuously smoking groups was $5.9 \mathrm{~kg}$ (95\% CI 4.9 to 7.0), $3.1 \mathrm{~kg}$ (CI 1.1 to 5.0), and $1.1 \mathrm{~kg}$ (CI 0.5 to 1.5$)$, respectively $(\mathrm{p}<0.05)$. Smoking status, education level $(\mathrm{p}=0.04)$, and baseline weight $(\mathrm{p}=0.01)$ were associated with initial weight gain. Sex, age, amount smoked, CO concentrations, and dietary restraint were not associated with initial weight gain. At 12 months, participants without a college degree gained more weight than those with a college degree (7.2 $\mathrm{kg} v 4.6 \mathrm{~kg}, \mathrm{p}=0.01)$.
\end{abstract}

\section{Conclusions}

Continuously abstinent individuals had a greater weight gain than continuously smoking and point prevalent abstinent individuals. Those with higher baseline weight and those with lower educational attainment had greater initial weight gain.

Sources of funding: National Heart, Lung, and Blood Institute and the state of Tennessee.

For article reprint: $\operatorname{Dr} R$ C Klesges, University of Memphis, Universities Prevention Center, 5350 Poplar Avenue, Suite 430, Memphis, TN 38119, USA. Fax +1 9017671350.

\section{Commentary}

This study by Klesges $e t$ al shows weight gain after smoking cessation among a group of people who continually abstained from smoking. The results challenge previous studies which may have underestimated weight gain after smoking cessation. The authors have highlighted the complex factors associated with health related behaviours. In particular, the study shows the dilemma health professionals face when making decisions to change health related behaviours, in this case, the benefit of smoking cessation compared with the cost of weight gain.

Strengths of this study include the inclusion of a point prevalent abstinence group (abstinent at the time data were collected but not continuously), participation of individuals in all 3 follow up sessions, and the confirmation of self reported smoking status by $\mathrm{CO}$ tests. The study could have been enhanced by detailed data collection regarding weight fluctuation history; dieting history; calorific intake before, during, and after the intervention; and exercise regimens, all of which may affect weight gain or loss. Given the extensive literature examining the relation between dietary restraint and obesity, ${ }^{1}$ it is surprising that in this study, dietary restraint was not related to initial weight gain. As the authors acknowledge, the study may have recruited a biased sample because those who chose to participate may not have been overly concerned with the possibility of weight gain after smoking cessation.

An interesting area for future study is the degree to which weight gain after smoking cessation can be accounted for by metabolic changes or changes in eating behaviour. This has important implications for the treatment of excessive weight gain $(\geqslant 9.1 \mathrm{~kg})$ after smoking cessation ( $16 \%$ in this sample).

In a culture preoccupied with physical appearance, nurses should be attentive to weight maintenance or weight gain as disincentives to stopping smoking, particularly for young women. The study findings substantiate the need to identify effective methods for reducing weight gain after quitting smoking, particularly in those with lower educational attainment.

Anne Garland RMN Clinical Research Associate Royal Victoria Infirmary Newcastle upon Tyne, UK

1 Williamson, DA. Assessment of eating disorders: obesity, anorexia, and bulimia nervosa. New York: Pergamon Press, 1990. 